\title{
How to build a human brain: Evolution, development, and education
}

\author{
Aaron P. Blaisdell, UCLA
}

At the 2018 annual EMG symposium held at Giessen, Germany, I presented a keynote talk of the same title as this extended abstract. I gave a similar talk at the Ancestral Health Symposium in 2014 (https://www.youtube.com/watch?v=gfhudq8J2Yc\&feature=youtu.be), and at invited symposia and in courses at UCLA. I have also previously published an article in the Journal of Evolution and Health on this topic (Blaisdell, 2015). What follows is an extended abstract of the EMG 2018 talk. It has been updated and condensed compared to the previous publication, with an emphasis on new material I have incorporated since the original publication appeared in print.

Let's begin with the question, what are brains for? Brains, and nervous systems in general, evolved to handle movement through space and time. This overarching goal requires three tightly linked and interacting functions. 1. The integration of sensory input to monitor the state of the world, both external and internal. 2. The organization of response output to solve adaptive decisions in that world. And 3. The operation of computational processes that encode, store, retrieve experiences and that operate on this information.

Based on computational prowess, humans are the smartest species on Earth. Why and how is our brain built to be so smart? Answers to these questions can be gleaned through comparisons to other species, especially our closest relatives the great apes. The evidence comes in three types: evolution, development, and learning. I will discuss each of these three lines of evidence in turn. The conclusion drawn from a careful analysis of all three branches of evidence leads us to an improved understanding of how and why humans are so smart relative to other species, and how we can optimize the conditions for peak performance.

Evolution by natural selection is the process that adapts an organism's traits to best function in the organism's environment. Evolutionary analysis includes both phylogenetic investigations, that is comparisons across species, and of the resulting adaptations selected by environments over evolutionary time. If humans are so smart, and intelligence is a function of the computational prowess of the brain, then what is special about the human brain to beget such species-unique intelligence? First, humans are primates, and compared to non-primate mammals, as cortical mass increases (in larger brained animals relative to smaller brained animals) the number of neurons in the primate cortex increases at a much higher rate than it does in the non-primate mammalian cortex (Herculano-Houzel, 2016). (Interestingly, birds follow a primate scaling rule!) The cortex is the outer surface of the mammalian brain and is involved in higher functions such as processing allocentric relations (relations between aspects of the world) and egocentric relations (self-world relations).

Also, in non-primate mammals, as cortical size increases so too do the size of its neurons. Not so in primates for which the neurons stay roughly the same size regardless of the size of the cortex (again, birds follow the primate pattern). Moreover, humans and some other primates have many more neurons in the cortex for every non-cortical neuron, a ratio of about 24 cortical neurons for every non-cortical neuron in the brain (Herculano-Houzel, 2016). Non-primate mammals, on the other hand, have a much lower ratio of cortical to non-cortical neurons, ranging from 2:1 (for shrews and moles) to 11:1 (for pigs, whales, and ruminants). So, one answer to the question of why are human brains so smart, is because we have an absolutely incredibly greater number of 
neurons in our cortex compared to other mammals, including the great apes, and even compared to the non-cortical part of our brains. More neurons, especially in cortex, begets more computing power (Garlick, 2010).

The larger human brain (three times as large as our nearest living relative, the chimpanzee), and larger, more neurally dense cortex, requires special adaptations and genetic changes for proper development prior to adulthood (a more detailed review of this evidence is presented in (Blaisdell, 2015). Some key signatures for special adaptations for brain development that are uniquely human include multiple duplication events for a gene (SRGAP2) that governs spinal density of neurons (Dennis et al., 2012). This gene regulates the generation of spines on dendrites of neurons. Dendrites are the branches extending from the cell body of a neuron that gather information from neighboring neurons for the purpose of decision making (i.e., whether or not to fire an action potential). Dendritic spines are the projections that extend from the dendrite that connect to other neurons. The neuron starts out with many more spines than are needed, and a pruning process pares down the number of spines as a function of experience, resulting in a more sparsely connected neuron (McShea \& Hordijk, 2013). These sparse neurons actually encode abstract representations derived from learning experiences, and thus the pruning process is key to developing smarter neurons and therefore smarter brains. Thus, by increasing the number of dendritic spines at the starting point, this allows for the pruning process to make much more intelligent brains that represent the world at much greater levels of abstraction than is possible in any other animal (Garlick, 2010).

Furthermore, compared to non-human primates, the human prefrontal cortex (PFC) has a 12-fold increase in the expression of human-unique genes (Liu et al., 2012). The PFC is a brain area involved in complex processes such as planning, cognitive flexibility, inhibition of impulsivity, guiding mental simulations (i.e., using your imagination), and counterfactual reasoning. The ratio of human-specific differences over chimpanzee-specific differences in genes governing lipid concentration changes has recently been found to be maximal not during early age, but in early adulthood (between 20-35 years of human-scaled age), suggesting functional rearrangements of the PFC lipidome unique to humans (Li et al., 2017; see also Bozek et al., 2015). Clearly the PFC is one of the primary sources of our intelligence. Not only are there many more human-unique genes expressed in the PFC compared to chimpanzees and macaque monkeys, the expression of these genes is delayed and extended during our lifetime compared to these other primates. Evolution often acts by tweaking developmental expression of genes and developmental programs, a process called heterochrony. The human PFC shows signatures of developmental heterochrony that suggest a novel phase of human ontogeny from 3-7 years of age. This coincides with a special window of development in cognitive maturity in humans, specifically self-regulation, abstract thinking, and social behavior (Thompson \& Nelson, 2011). Compared to chimpanzees and other apes, humans show an extended early childhood and adolescence. This extended development, including the human-unique period of early childhood (ages 4-6 years old) is likely what contributes to unique human cognitive traits, such as language, tool use, cooperation, and humor, as well as retention of juvenile traits characteristic of juvenile apes into human adulthood, such as playfulness, curiosity, and laughter, which can be considered cognitive neotenies.

Let's finally turn to education and intellectual development. While the field of developmental psychology has deep historical roots, it's only been in the past twenty years that developmental psychologists have turned their attention on the processes by which human infants develop intelligence and cognition. What is emerging from this new focus is the view of infants as rational 
constructivist learnings (Gopnik \& Wellman, 2012; Xu \& Kushnir, 2013). What this means is that, rather than being passive learners starting from a blank slate, infants come to the world equipped with predispositions and prior beliefs about how the world should work, such as causal (Lu \& Cheng, 2017) and perceptual (Wood, 2013) invariance. Furthermore, infants are active consumers of data and probe the world through experiments and exploration to discover world knowledge and test their nascent theories and beliefs. In short, early learning is viewed as being rational, statistical, and inferential (Xu and Kushnir, 2013). Research from this perspective is finding that free play can be just as good as directed instruction in guiding learning. In an experiment by (Sim \& Xu, 2014), for example, 3 year olds were either shown how toy blocks activate a machine to make a sound, or they were allowed to investigate the blocks and machine through free play. When subsequently asked to choose novel blocks to make a familiar or novel machine make a sound, infants given free-play alone were just as accurate as trained kids at making inferences about machine function.

There is growing recognition that the ability of free play to guide intellectual and cognitive development is an adaptation to training the human brain to be smart (Blaisdell, 2015; (Buchsbaum et al., 2012). Bucshbaum et al. write "We hypothesize that the change in the developmental program that led to the uniquely long period of human childhood allowed immature protohumans to enjoy longer protected periods of learning and, in particular, to engage more extensively in the free exploration found in play." This hypothesis is consistent with the lines of evidence from brain evolution and development that I laid out above and previously (Blaisdell, 2015). Buchsbaum et al. specifically single out pretend and symbolic play as a uniquely human form of play that is critical for intellectual development.

"Human children, unlike any other immature animal, engage in a particularly distinctive kind of pretend or symbolic play." "Children go beyond simply practicing actions they will require later or manipulating objects to discover their causal features." "Instead, they work out quite elaborate unreal scenarios, often with the aid of language, props and gestures. Investment in an extended childhood, with its many opportunities for free exploration and causal learning, may have allowed human beings to turn from simply making the same ecological widgets to developing our staggeringly wide variety of strategies for adaptive success."

This advanced form of play evolved as an adaptation to build large, intelligent human brains capable of solving complex tasks involving tool-use, language, culture, and prosocial, cooperative social systems over the course of human evolution. If life in a hunter-gather band society allows for proper development of human intellect (and the ancestral human ecological niche has been suggested to be the driver of human brain evolution (González-Forero \& Gardner, 2018)), then the question arises, does our modern form of schooling and education also meet the needs of optimal intellectual development? We must ask this question because the modern educational system is so different, so far removed from the ancestral environment of child development and intellectual growth, that the possibility of evolutionary mismatch is significant. If we can identify such evolutionary mismatches and their consequences, then perhaps we can do something to address them and foster better, if not optimal child development and intellectual growth in modern society.

There are a number of examples that directed instruction in a school setting can impede or even erode intellectual development. In a study by Kamii and Dominick (1997), $2^{\text {nd }}, 3^{\text {rd }}$, and $4^{\text {th }}$ graders 
were tested for effects of teaching computational algorithms such as those of "carrying." Some children had been encouraged to invent their own procedures and had not been taught any algorithms from grades 1 to 2 or 3 . Others had been taught the conventional algorithms prescribed by textbooks. Both groups of children where then asked to solve multi-digit addition and multiplication problems and asked to explain how they got their answers. Contrary to expectations based on the value of directed instruction, the children with no directed instruction were more accurate $(45 \%$ correct) than the directed instruction group (12\% correct). More telling were the errors. When the children without directed instruction were wrong, their incorrect answers were clustered closely around the correct answer. That is, they were off by a small amount, indicating that they understood the problem and how to solve it and made small math errors in their estimation or calculations. In dramatic contrast, the children that had received instruction on algorithms made errors that in many cases were wildly off base. For example, when solving the equation ' $7+52+186$ ', while only $12 \%$ of the directed instruction children got the correct answer of '245', many children came up with answers like '29' or '30' on the very low end, or '1000' or '9308' at the very high end of the range. Kamii and Dominick concluded that directed instruction on algorithms "untaught" the children's earlier acquired conception of 'place value' and number sense.

Ashton (1975) reviewed evidence that understanding of conservation of physical properties, such as that when water from a short cylinder is poured into a tall cylinder the volume of water does not change, is delayed or declined in children receiving too much or overly restrictive formal schooling. Similarly, a more recent study found that the amount of time children spent in less structured activities (free play, self-initiated practice, reading, social outings, etc.) correlated positively with verbal fluency, a measure of self-directed executive functioning (PFC function such as self-regulation and advanced cognition), while children who reported spending more time on more structured activities (lessons, homework, tutoring, chores, and formal organizational meetings) showed lower verbal fluency scores (Barker, Semenov, Michaelson, Provan, \& Snyder, 2014).

Evolutionary Psychologist Peter Gray has perhaps been the staunchest advocate for pointing out the harms of academic training, particularly in early childhood. Despite having initial academic advantages, directed academic training has been shown to have no lasting effect on academic performance in young children (Gray, 2015a). Children in more play-based preschools catch up and surpass by grade 4 the academic performance of children that attended more academicallyfocused preschools.

Perhaps more important are the effects of academic focused early childhood education programs on mental health and wellbeing. For example, exposure to a direct-instruction preschool program was also associated with later outcomes of higher rates of violence than was exposure to a playbased preschool program (Gray, 2015b). Gray (2015b) argues that teaching academic skills to children before they have developed the requisite motivational and intellectual foundations can do more harm than good. He suggests this harm stems from both the lack of motivation to learn academic skills before the child understands them, and because academic skills are necessarily procedure-based, and that the procedures won't consolidate (form) into long-term memory if they have no meaning to the child. Similar detrimental effects of cognitive training introduced too early during development have shown to harm adult cognition in animal models, as well. For example, Harlow (1959) trained Rhesus monkeys trained on an object-discrimination procedure. For different groups of monkeys, training began at ages of Post Natal Day (PND) 60, 90, 120, or 150. 
By PND 250, there was no evidence for discrimination learning in the monkeys that had begun training below PND 90. When they reached adulthood, the monkeys that had started discrimination training below PND 90 also showed poor set learning, a task that requires a wellfunctioning PFC. Thus, training introduced when the individual is not developmentally ready for it can impair development of later learning potential, perhaps through interfering with the developmental process itself. Similar results in rats have been reported by Spear and Hyatt (1993).

The detrimental effect on cognition of introducing cognitive training before the individual is developmentally ready suggests a process of decanalization, where by the developmental trajectory is thrown off course by the training, shifting cognitive development away from its normal, optimal path and towards an alternative, suboptimal path. This decanalization model of developmental mismatches has been applied to explain many human psychopathologies, ranging from schizophrenia and attention-deficit, hyperactivity disorder (ADHD; McGrath, Hannan, \& Gibson, 2011), to mood disorders and impaired intellectual/cognitive development (Blaisdell, 2015).

Rather than focusing on academic training, Gray argues that by allowing children to first engage in self-directed and self-motivated play and exploration, they will form the intellectual skills of knowledge, understanding, and analytic cognition. Once these skills have formed, it is only then that instruction in academic skills can be successful. For example, the evidence from Kamii and Dominick (1997) that academic training of mathematical algorithms impairs and erodes children's understanding of place value and number sense should not be taken to imply that such training is inherently bad. Rather, such training should not be introduced too early when the individual is not developmentally ready to benefit from such training, but should be introduced at later ages when the individual is developmentally ready to benefit from it. Clearly, academic training is highly useful and functional for adapting us optimally to our modern information-based society. Yet, the caution being advocated here is to allow the child sufficient time to develop using the original human adaptation of free play and exploration, along with being immersed in a prosocial, interactive culture, listening to stories and observing and interacting with many other children of all ages and adults, before introducing increasingly rigorous academic training to bolster rather than hinder or erode intellectual growth.

Like hunter-gatherer band society, modern society must allow the production of individuals who are creative, analytic, innovative, and generalist problem solvers, with well-functioning brains, minds, and emotions. How do we achieve this? Take advantage of the tools that adaptation has outfitted the human brain to train itself and guide its own development in educational and developmental settings that mimic the physical and social contexts of small-band society. Return to play and exploration as children's natural ability to educate themselves, with a gradual supplementation with directed instruction and formalized, procedural skills when they reach developmental readiness, tailored to the individual (given non-linear development creates vast individual differences in rates of development). Children are born with adaptations to act like scientists and philosophers, artists and entrepreneurs. Let's design our educational systems to bolster these adaptations rather than suppress them. 


\section{References}

Ashton, P. T. (1975). Cross-cultural Piagetian research: An experimental perspective. Harvard Educational Review, 45(4), 475-506. https://doi.org/10.17763/haer.45.4.kpq1578t13tk0541

Barker, J. E., Semenov, A. D., Michaelson, L., Provan, L. S., Snyder, H. R., \& Munakata, Y. (2014). Less-structured time in children's daily lives predicts self-directed executive functioning. Frontiers in Psychology, 5, 593. https://doi.org/10.3389/fpsyg.2014.00593

Blaisdell, A. P. (2015). Play as the Foundation of Human Intelligence: The Illuminating Role of Human Brain Evolution and Development and Implications for Education and Child Development. Journal of Evolution and Health, 1(1), 1-54.

Bozek, K., Wei, Y., Yan, Z., Liu, X., Xiong, J., Sugimoto, M., ... \& Ely, J. J. (2015). Organization and evolution of brain lipidome revealed by large-scale analysis of human, chimpanzee, macaque, and mouse tissues. Neuron, 85(4), 695-702. https://doi.org/10.1016/j.neuron.2015.01.003

Buchsbaum, D., Bridgers, S., Weisberg, D. S., \& Gopnik, A. (2012). The power of possibility: Causal learning, counterfactual reasoning, and pretend play. Philosophical Transactions of the Royal Society of London B: Biological Sciences, 367(1599), 2202-2212. https://doi.org/10.1098/rstb.2012.0122

Dennis, M. Y., Nuttle, X., Sudmant, P. H., Antonacci, F., Graves, T. A., Nefedov, M., ... Eichler, E. E. (2012). Evolution of Human-Specific Neural SRGAP2 Genes by Incomplete Segmental Duplication. Cell, 149(4), 912-922. https://doi.org/10.1016/j.cell.2012.03.033

Garlick, D. (2010). Intelligence and the brain: Solving the mystery of why people differ in IQ and how a child can be a genius. Burbank, California: AESOP Press.

González-Forero, M., \& Gardner, A. (2018). Inference of ecological and social drivers of human brain-size evolution. Nature, 557(7706), 554. https://doi.org/10.1038/s41586-018-0127-x

Gopnik, A., \& Wellman, H. M. (2012). Reconstructing constructivism: causal models, Bayesian learning mechanisms, and the theory theory. Psychological Bulletin, 138(6), 1085-1108. https://doi.org/10.1037/a0028044

Gray, P. (2015a). Early academic training produces long-term harm. Psychology Today. Retrieved from https://www.psychologytoday.com/blog/freedomlearn/\%0A201505/earlyacademic-training-produces-long-term-harm

Gray, P. (2015b). How early academic training retards intellectual development. Psychology Today. Retrieved from https://www.psychologytoday.com/blog/freedom-learn/201506/howearlyacademic-\%0Atraining-retards-intellectual-development

Harlow, B. Y. H. F. (1959). Learning set and error factor theory. In S. Koch (Ed.), Psychology: A study of a science, Vol. 2.

Herculano-Houzel, S. (2016). The human advantage. MIT Press.

Kamii, C., \& Dominick, A. (1997). To teach or not to teach algorithms. Journal of Mathematical Behavior, 16(1), 51-61. https://doi.org/10.1016/S0732-3123(97)90007-9 
Li, Q., Bozek, K., Xu, C., Guo, Y., Sun, J., Pääbo, S., ... \& Willmitzer, L. (2017). Changes in lipidome composition during brain development in humans, chimpanzees, and macaque monkeys. Molecular biology and evolution, 34(5), 1155-1166. https://doi.org/10.1093/molbev/msx065

Liu, X., Somel, M., Tang, L., Yan, Z., Jiang, X., Guo, S., ... \& Li, N. (2012). Extension of cortical synaptic development distinguishes humans from chimpanzees and macaques. Genome Research, 22, 611-622. https://doi.org/10.1101/gr.127324.111

Lu, H., \& Cheng, P. W. (2017). Causal invariance as an essential constraint for creating a causal representation of the world: Generalizing the invariance of causal power. In M. R. Waldmann (Ed.), The Oxford Handbook of Causal Reasoning (p. 65).

McGrath, J. J., Hannan, a J., \& Gibson, G. (2011). Decanalization, brain development and risk of schizophrenia. Translational Psychiatry, 1(6), e14. https://doi.org/10.1038/tp.2011.16

Sim, Z., \& Xu, F. (2014, January). Acquiring inductive constraints from self-generated evidence. In Proceedings of the Annual Meeting of the Cognitive Science Society (Vol. 36, No. 36).

Spear, N., \& Hyatt, L. (1993). How the timing of experience can affect the ontogeny of learning. In G. Turkewitz \& D. A. Devenny (Eds.), Developmental Time and Timing (pp. 167-209).

Thompson, J. L., \& Nelson, A. J. (2011). Middle childhood and modern human origins. Human Nature, 22(3), 249. https://doi.org/10.1007/s12110-011-9119-3

Wood, J. N. (2013). Newborn chickens generate invariant object representations at the onset of visual object experience. Proceedings of the National Academy of Sciences, 110(34), 14000-14005. https://doi.org/10.1073/pnas.1308246110

Xu, F., \& Kushnir, T. (2013). Infants are rational constructivist learners. Current Directions in Psychological Science, 22(1), 28-32. Ashton, P. (1975). Cross-cultural Piagetian Research: An Experimental Perspective. Harvard Educational Review. https://doi.org/10.17763/haer.45.4.kpq1578t13tk0541

Barker, J. E., Semenov, A. D., Michaelson, L., Provan, L. S., \& Snyder, H. R. (2014). Lessstructured time in children ' $s$ daily lives predicts self-directed executive functioning, 5(June), 1-16. https://doi.org/10.3389/fpsyg.2014.00593

Blaisdell, A. P. (2015). Play as the Foundation of Human Intelligence: The Illuminating Role of Human Brain Evolution and Development and Implications for Education and Child Development. Journal of Evolution and Health, 1(1), 1-54.

Bozek, K., Wei, Y., Yan, Z., Liu, X., Xiong, J., Sugimoto, M., ... Khaitovich, P. (2015). Organization and Evolution of Brain Lipidome Revealed by Large-Scale Analysis of Human, Chimpanzee, Macaque, and Mouse Tissues. Neuron. https://doi.org/10.1016/j.neuron.2015.01.003

Buchsbaum, D., Bridgers, S., Weisberg, D. S., Gopnik, A., B, P. T. R. S., Buchsbaum, D., ... Gopnik, A. (2012). The power of possibility: causal learning, counterfactual reasoning, and pretend play, 2202-2212. https://doi.org/10.1098/rstb.2012.0122

Dennis, M. Y., Nuttle, X., Sudmant, P. H., Antonacci, F., Graves, T. A., Nefedov, M., ... Eichler, E. E. (2012). Evolution of Human-Specific Neural SRGAP2 Genes by Incomplete Segmental Duplication. Cell, 149(4), 912-922. https://doi.org/10.1016/j.cell.2012.03.033

Garlick, D. (2010). Intelligence and the brain: Solving the mystery of why people differ in IQ and 
how a child can be a genius. Burbank, California: AESOP Press.

González-Forero, M., \& Gardner, A. (2018). Inference of ecological and social drivers of human brain-size evolution. Nature. https://doi.org/10.1038/s41586-018-0127-x

Gopnik, A., \& Wellman, H. M. (2012). Reconstructing constructivism: causal models, Bayesian learning mechanisms, and the theory theory. Psychological Bulletin, 138(6), 1085-1108. https://doi.org/10.1037/a0028044

Gray, P. (2015a). Early academic training produces long-term harm. Psychology Today. Retrieved from https://www.psychologytoday.com/blog/freedomlearn/\%0A201505/earlyacademic-training-produces-long-term-harm

Gray, P. (2015b). How early academic training retards intellectual development. Psychology Today. Retrieved from https://www.psychologytoday.com/blog/freedom-learn/201506/howearlyacademic-\%0Atraining-retards-intellectual-development

Harlow, B. Y. H. F. (1959). Learning set and error factor theory. In S. Koch (Ed.), Psychology: A study of a science, Vol. 2.

Herculano-Houzel, S. (2016). The human advantage. MIT Press.

Kamii, C., \& Dominick, A. (1997). To teach or not to teach algorithms. Journal of Mathematical Behabior. https://doi.org/10.1016/S0732-3123(97)90007-9

Li, Q., Bozek, K., Xu, C., Guo, Y., Sun, J., Pääbo, S., ... Khaitovich, P. (2017). Changes in Lipidome Composition during Brain Development in Humans, Chimpanzees, and Macaque Monkeys. Molecular Biology and Evolution. https://doi.org/10.1093/molbev/msx065

Liu, X., Somel, M., Tang, L., Liu, X., Somel, M., Tang, L., .. Pa, S. (2012). from chimpanzees and macaques Extension of cortical synaptic development distinguishes humans from chimpanzees and macaques, 611-622. https://doi.org/10.1101/gr.127324.111

Lu, H., \& Cheng, P. W. (2017). Causal invariance as an essential constraint for creating a causal representation of the world: Generalizing the invariance of causal power. In M. R. Waldmann (Ed.), The Oxford Handbook of Causal Reasoning (p. 65).

McGrath, J. J., Hannan, a J., \& Gibson, G. (2011). Decanalization, brain development and risk of schizophrenia. Translational Psychiatry, 1(6), e14. https://doi.org/10.1038/tp.2011.16

McShea, D. W., \& Hordijk, W. (2013). Complexity by Subtraction. Evolutionary Biology, 40(4), 504-520. https://doi.org/10.1007/s11692-013-9227-6

Sim, Z. L., \& Xu, F. (2014). Acquiring inductive constraints from self-generated evidence. In Proceedings of the 36th Annual Conference of the Cognitive Science Society.

Spear, N., \& Hyatt, L. (1993). how the timing of experience can affect the ontogeny of learning. In G. Turkewitz \& D. A. Devenny (Eds.), Developmental Time and Timing (pp. 167-209).

Thompson, J. L., \& Nelson, A. J. (2011). Middle Childhood and Modern Human Origins, (August), 249-280. https://doi.org/10.1007/s12110-011-9119-3

Wood, J. N. (2013). Newborn chickens generate invariant object representations at the onset of visual object experience. Proceedings of the National Academy of Sciences. https://doi.org/10.1073/pnas.1308246110 
Xu, F., \& Kushnir, T. (2013). Infants Are Rational Constructivist Learners. Current Directions in Psychological Science. https://doi.org/10.1177/0963721412469396 